\title{
ON A FAMILY OF LOGARITHMIC AND EXPONENTIAL INTEGRALS OCCURRING IN PROBABILITY AND RELIABILITY THEORY
}

\author{
M. ASLAM CHAUDHRY ${ }^{1}$
}

(Recieved 26 May 1992; revised 6 February 1993)

\begin{abstract}
We define an integral function $I_{\mu}(\alpha, x ; a, b)$ for non-negative integral values of $\mu$ by

$$
\begin{aligned}
I_{\mu}(\alpha, x ; a, b)=\int_{x}^{\infty}(\ln t)^{\mu} t^{\alpha-1} \exp \left(-a t-b t^{-1}\right) d t \\
\quad(x \geq 0, a>0, b>0,-\infty<\alpha<\infty) .
\end{aligned}
$$

It is proved that $I_{\mu}(\alpha, x ; a, b)$ satisfies a functional recurrence relation which is exploited to find a closed form evaluation of some incomplete integrals. New integral representations of the exponential integral and complementary error functions are found as special cases.
\end{abstract}

\section{Introduction}

The inverse Gaussian density function, which arises as the density of the first passage time of the Brownian motion with positive drift, is given by

$$
f_{T}(t)=\left(v / 2 \pi t^{3}\right)^{1 / 2} \exp \left(-v(t-\mu)^{2} / 2 \mu^{2} t\right), \quad t, v, \mu>0 .
$$

The model (1) has been used in the statistical theory of demographic rates and in reliability theory. (see [6], [7], [13], [14], [16], [17], [19], [20]). Good [10] proposed the generalized inverse Guassian distribution

$$
\begin{aligned}
& f(t)=\frac{1}{I_{0}(\alpha ; a, b)} t^{\alpha-1} \exp \left(-a t-b t^{-1}\right), \\
&(t>0, a>0, b>0,-\infty<\alpha<\infty),
\end{aligned}
$$

\footnotetext{
${ }^{1}$ Dept of Math. Sciences, King Fahd Univ. of Petroleum and Minerals, Dhahran, Saudi Arabia. (C) Australian Mathematical Society, 1994, Serial-fee code 0334-2700/94
} 
where

$$
\begin{gathered}
I_{0}(\alpha ; a, b)=\int_{0}^{\infty} t^{\alpha-1} \exp \left(-a t-b t^{-1}\right) d t=2(b / a)^{\alpha / 2} K_{\alpha}(2 \sqrt{a b}) \\
(a>0, b>0) .
\end{gathered}
$$

Some of the probabilistic properties of the distribution (2) were investigated in [4], [6], [10], [14], [19], [20].

Sichel [17] used (2) to construct mixtures of Poisson distributions and Barndorff-Nielson [4] used it to obtain the generalized hyperbolic distribution as a mixture of normal distributions. Chaudhry and Ahmad [1] derived the model (2) as a solution to a dynamical system in catastrophe theory. It was shown that several classical densities such as the Weibull, Gamma, Erlang, Exponential, Raleigh, Chi-square and Log-normal can be derived from (2) by simple transformations of the variable $t$ or by specializing the parameters, $\alpha, a$ and $b$. This provided a unified approach to the systematic study of the probability density functions encountered in probability and reliability theory.

In the course of describing some statistical properties of (2), it was needed as well to evaluate the incomplete integral

$$
\begin{aligned}
I_{\mu}(\alpha, x ; a, b)=\int_{x}^{\infty}(\ln t)^{\mu} t^{\alpha-1} \exp \left(-a t-b t^{-1}\right) d t \\
\quad(x \geq 0, a>0, b>0,-\infty<\alpha<\infty),
\end{aligned}
$$

for non-negative integral values of $\mu$.

An extensive search of the literature was done (see [5], [8], [9], [11], [12], [18]). It was found that $I_{\mu}(\alpha, x ; a, b)$ is known only when $x=0, \mu=0$, [11, page $340(3.471)(9)]$. The problem still remains open and the search is continued. However, during this search we were able to evaluate another allied incomplete integral

$$
\int_{x}^{\infty}(\ln t) t^{\alpha-1}\left(a-\frac{\alpha}{t}-\frac{b}{t^{2}}\right) \exp \left(-a t-b t^{-1}\right) d t
$$

$(x>0, a>0, b>0,-\infty<\alpha<\infty)$, for $\alpha=n+\frac{1}{2}, n=0, \pm 1, \pm 2, \pm 3, \ldots$. To the best of our knowledge, the closed form solution to (4) does not seem to be known for any value of $\alpha$ for $x>0$. For $x=0$, (4) has recently been solved by Chaudhry and Ahmad [2] for all $\alpha$, which extended the earlier results known only for $\alpha=n+\frac{1}{2}, n=0, \pm 1, \pm 2, \pm 3, \ldots 0$ (see [11, pages 577-578]). As a 
corollary to our result, we find a new integral representation

$$
\frac{1}{a} E_{i}(-a x)=(\ln x) \exp (-a x)-\int_{x}^{\infty}(\ln t) \exp (-a t) d t \quad(x>0, a>0),
$$

of the exponential integral function. The new representation (5) is extremely useful in the study of the well-testing phenomenon when the pressure solution of the diffusivity equation,

$$
\frac{1}{r} \frac{\partial}{\partial r}\left(\frac{p}{\mu z} r \frac{\partial p}{\partial r}\right)=\frac{\phi}{0.000264 k} \frac{\partial}{\partial r}\left(\frac{p}{z}\right),
$$

is expressed in terms of the exponential integral function as a function of two parameters viz. time and distance (see [15] and the references cited in it).

It should be noted that (5) extends the known result [11, page 926(8.212)(16)]

$$
E_{i}(-a)=-a \int_{1}^{\infty}(\ln t) \exp (-a t) d t, \quad a>0 .
$$

We have also obtained a new integral representation

$$
\operatorname{Erfc}(x)=\frac{x}{\sqrt{\pi}} \int_{1}^{\infty}(\ln t)\left[\frac{2 t x^{2}-1}{2 \sqrt{t}}\right] \exp \left(-t x^{2}\right) d t, \quad x>0,
$$

of the complementary error function as a special case of our result.

It is anticipated that the present work will be useful in different fields of engineering, probability and reliability theory.

THEOREM 1. Let $I_{\mu}(\alpha, x ; a, b)$ be defined as in (3). Then

$$
\begin{array}{r}
a I_{\mu}(\alpha+1, x ; a, b)=\alpha I_{\mu}(\alpha, x ; a, b)+b I_{\mu}(\alpha-1, x ; a, b) \\
+\mu I_{\mu-1}(\alpha, x ; a, b)+(\ln x)^{\mu} x^{\alpha} \exp \left(-a x-b x^{-1}\right) \\
(x>0, a>0, b>0,-\infty<\alpha<\infty) .
\end{array}
$$

ProOF. For $0<t<\infty$, let $F(t)$ be defined by

$$
F(t)=(\ln t)^{\mu} t^{\alpha} \exp \left(-a t-b t^{-1}\right), \quad a>0, b>0,-\infty<\alpha<\infty .
$$

Since $\alpha t-a t^{2}+b \leq 0$ as $t \rightarrow \infty$, it follows that for sufficiently large $t$

$$
\frac{d F}{d t}=t^{\alpha-2}(\ln t)^{\mu-1}\left\{\left(\alpha t-a t^{2}+b\right) \ln t+\mu t\right\} \exp \left(-a t-b t^{-1}\right) \leq 0
$$


for all $a>0, b>0$ and $-\infty<\alpha<\infty$. It is, therefore, allowable to apply the generalized mean value theorem (see [5], [12]) to the integral $\int_{x}^{\infty} F^{\prime}(t) d t$, $x>0$. However $\lim _{t \rightarrow \infty} F(t)=0$; so we have

$$
\int_{x}^{\infty} F^{\prime}(t) d t=0-F(x), \quad x>0 .
$$

Substituting the value of $F^{\prime}(t)$ and $F(x)$ in (11) we obtain

$$
\begin{array}{r}
\int_{x}^{\infty}(\ln t)^{\mu}\left\{\alpha t^{\alpha-1}-a t^{\alpha}+b t^{\alpha-2}\right\} \exp \left(-a t-b t^{-1}\right) d t \\
+\mu \int_{x}^{\infty}(\ln t)^{\mu-1} t^{\alpha-1} \exp \left(-a t-b t^{-1}\right) d t \\
=-(\ln x)^{\mu} x^{\alpha} \exp \left(-a t-b t^{-1}\right)
\end{array}
$$

which, after simplification, proves the theorem.

REMARK. If $x>0$, (8) holds true for all $a>0, b \geq 0$ and $-\infty<\alpha<\infty$. However, if we take $x=0$, then, using the fact that

$$
\lim _{t \rightarrow 0^{+}} F(t)=0
$$

we get

$$
\begin{gathered}
a I_{\mu}(\alpha+1,0 ; a, b)=\alpha I_{\mu}(\alpha, 0 ; a, b)+b I_{\mu}(\alpha-1,0 ; a, b)+\mu I_{\mu-1}(\alpha, 0 ; a, b) \\
(a>0, b>0,-\infty<\alpha<\infty) .
\end{gathered}
$$

COROLlARY. (See [2] and [11, pages 577-578])

$$
\int_{0}^{\infty}(\ln t) t^{\alpha}\left\{a-\frac{\alpha}{t}-\frac{b}{t^{2}}\right\} \begin{gathered}
\exp \left(-a t-b t^{-1}\right) d t=2(b / a)^{\alpha / 2} K_{\alpha}(2 \sqrt{a b}) \\
(a>0, b>0,-\infty<\alpha<\infty)
\end{gathered}
$$

PROOF. This follows from (13) when we substitute $\mu=1$ and replace $I_{1}(\alpha-$ $1,0 ; a, b)$ and $I_{1,0}(\alpha, 0 ; a, b)$ by their integral representations. In particular, when $\alpha=0$ in (14) we get [11, page 577(4.356)(4)]

$$
\int_{0}^{\infty}(\ln t)\left\{\frac{a t^{2}-b}{t^{2}}\right\} \exp \left(-a t-b t^{-1}\right) d t=2 K_{0}(2 \sqrt{a b}), \quad a>0, b>0 .
$$


It should be noted that the results [11, pages 577-578(4.356)(1-6)] and [11, page $578(4.359)(1-3)]$ are special cases of (14) for different values of the parameters $\alpha, a$ and $b$.

\section{THEOREM 2.}

$$
\begin{aligned}
& \int_{x}^{\infty}(\ln t) t^{1 / 2}\left[a-\frac{1}{2 t}-\frac{b}{t^{2}}\right] \exp \left(-a t-b t^{-1}\right) d t \\
& =\frac{1}{2} \sqrt{\frac{\pi}{a}}\left[\exp (-2 \sqrt{a b}) \operatorname{Erfc}\left(\sqrt{a x}-\sqrt{\frac{b}{x}}\right)+\exp (2 \sqrt{a b}) \operatorname{Erfc}\left(\sqrt{a x}+\sqrt{\frac{b}{x}}\right)\right] \\
& +(\ln x) x^{1 / 2} \exp \left(-a x-b x^{-1}\right) \\
& (x>0, a>0, b>0) \text {. }
\end{aligned}
$$

PROOF. Substituting $\mu=1$ and $\alpha=\frac{1}{2}$ in (8) we get

$$
\begin{aligned}
& a I_{1}\left(\frac{3}{2}, x ; a, b\right)-\frac{1}{2} I_{1}\left(\frac{1}{2}, x ; a, b\right)-b I_{1}\left(-\frac{1}{2}, x ; a, b\right) \\
&= I_{0}\left(\frac{1}{2}, x ; a, b\right)+(\ln x) x^{1 / 2} \exp \left(-a x-b x^{-1}\right) \\
&(x>0, a>0, b>0,-\infty<\alpha<\infty) .
\end{aligned}
$$

It follows from (17) that we need to evaluate $I_{0}\left(\frac{1}{2}, x ; a, b\right)$ only. However, since

$$
I_{0}\left(\frac{1}{2}, x ; a, b\right)=a^{-1 / 2} I_{0}\left(\frac{1}{2}, a x ; 1, a b\right),
$$

we solve

$$
I(x)=\int_{x}^{\infty} t^{-1 / 2} \exp \left(-t-b t^{-1}\right) d t, \quad x>0 .
$$

We note that

$$
I\left(\frac{1}{x}\right)=\int_{1 / x}^{\infty} t^{-1 / 2} \exp \left(-t-b t^{-1}\right) d t .
$$

Substituting $t=1 / \tau$ in (20) we get

$$
I\left(\frac{1}{x}\right)=e^{-b x}\left(\left\{e^{b x}\right\} *\left\{x^{-3 / 2} e^{-1 / x}\right\}\right)
$$

where '*' is the convolution operator defined by

$$
a(x) * b(x)=\int_{0}^{x} a(x-t) b(t) d t
$$


We can write (20) in the operational form (see [9, page 131(20) and page 246(10)])

$$
I(x)=e^{-b x} L^{-1}\left\{\frac{1}{p-b} e^{-2 \sqrt{p}} ; \frac{1}{x}\right\},
$$

where $L^{-1}$ is the inverse Laplace transform operator.

Using the identity [9, page 246(10)] we get

$$
I(x)=\frac{1}{\sqrt{\pi}}\left[\exp (-2 \sqrt{b}) \operatorname{Erf} c\left(\sqrt{x}-\sqrt{\frac{b}{x}}\right)+\exp (2 \sqrt{b}) \operatorname{Erfc}\left(\sqrt{x}+\sqrt{\frac{b}{x}}\right)\right] .
$$

From $(18)-(22)$ we get

$$
\begin{array}{r}
I_{0}\left(\frac{1}{2}, x ; a, b\right)=\frac{1}{2} \sqrt{\frac{\pi}{a}}\left[\exp (-2 \sqrt{a b}) \operatorname{Erfc}\left(\sqrt{a x}-\sqrt{\frac{b}{x}}\right)\right. \\
\left.+\exp (2 \sqrt{a b}) \operatorname{Erfc}\left(\sqrt{a x}+\sqrt{\frac{b}{x}}\right)\right] \\
(x>0, a>0, b>0) .
\end{array}
$$

Replacing $I_{1}\left(\frac{3}{2}, x ; a, b\right), \quad I_{1}\left( \pm \frac{1}{2}, x ; a, b\right)$ by their integral representations and substituting the value of $I_{0}\left(\frac{1}{2}, x ; a, b\right)$ from (23) in (17) proves Theorem 2.

The following corollary is the consequence of (16) and does not seem to be known in the literature.

COROLlaRY.

$$
\begin{gathered}
\int_{1}^{\infty}(\ln t) t^{1 / 2}\left[a-\frac{1}{2 t}-\frac{b}{t^{2}}\right] \exp \left(-a t-b t^{-1}\right) d t \\
=\frac{1}{2} \sqrt{\frac{\pi}{a}}[\exp (-2 \sqrt{a b}) \operatorname{Erfc}(\sqrt{a}-\sqrt{b})+\exp (2 \sqrt{a b}) \operatorname{Erfc}(\sqrt{a}+\sqrt{b})] \\
\quad(a>0, b>0) .
\end{gathered}
$$

PROOF. This follows from (16) when we take $x=1$. In particular, when we take $b=a$ in (24), we get

$$
\begin{aligned}
\int_{1}^{\infty}(\ln t) t^{1 / 2} & {\left[a-\frac{1}{2 t}-\frac{a}{t^{2}}\right] \exp \left(-a\left(t+t^{-1}\right)\right) d t } \\
& =\frac{1}{2} \sqrt{\frac{\pi}{a}} \exp (-2 a)[1+\exp (4 a) \operatorname{Erfc}(2 \sqrt{a})], \quad a>0 .
\end{aligned}
$$


REMARK. It can be seen that

$$
\frac{\partial}{\partial b}\left\{I_{0}(\alpha, x ; a, b)\right\}=-I_{0}(\alpha-1, x ; a, b), \quad a>0, b>0 .
$$

Therefore, it follows from (8), (17) and (26) that we can evaluate the semi-infinite integral

$$
\int_{x}^{\infty}(\ln t) t^{\alpha}\left[a-\frac{\alpha}{t}-\frac{b}{t^{2}}\right] \exp \left(-a t-b t^{-1}\right) d t,
$$

for $\alpha=n+\frac{1}{2}, n=0, \pm 2, \pm 3, \ldots$, in terms of complementary error functions. For other values of $\alpha$ the problem remains open.

\section{THEOREM 3.}

$$
\begin{gathered}
\int_{x}^{\infty}(\ln t) t^{\alpha}[a t-\alpha] \exp (-a t) \frac{d t}{t}=a^{-\alpha} \Gamma(\alpha, a x)+(\ln x) x^{\alpha} \exp (-a x) \\
(x>0, a>0)
\end{gathered}
$$

PROOF. Since $x>0$, we substitute $b=0$ and $\mu=1$ in (8) to get

$$
\begin{gathered}
a I_{1}(\alpha+1, x ; a, 0)-\alpha I_{1}(\alpha, x ; a, 0)=I_{0}(\alpha, x ; a, 0)+(\ln x) x^{\alpha} \exp (-a x) \\
(x>0, a>0) .
\end{gathered}
$$

However (see [11, page 317(3.381)(3)])

$$
I_{0}(\alpha, x ; a, 0)=a^{-\alpha} \Gamma(\alpha, a x), \quad a>0 .
$$

Replacing $I_{1}(\alpha+1, x ; a, 0), \quad I_{1}(\alpha, x ; a, 0)$ by their integral representations in (28) and using (29) we get the proof of the theorem.

That the following corollaries are the consequence of (27) does not seem to be known in the literature.

COROLLARY 1.

$$
\begin{aligned}
& \operatorname{Erfc}(\sqrt{a x})=\sqrt{\frac{a}{\pi}}\left[\int_{x}^{\infty}(\ln t)\left[\frac{2 a t-1}{2 \sqrt{t}}\right] \exp (-a t) d t-(\ln x) \sqrt{x} \exp (-a x)\right] \\
& (x>0, a>0) \text {. }
\end{aligned}
$$


ProOF. This follows from (27) when we take $\alpha=\frac{1}{2}$ and use the fact that $\Gamma\left(\frac{1}{2}, z^{2}\right)=\sqrt{\pi} \operatorname{Erfc}(z)$. In particular when we take $x=1$ and replace $a$ by $z^{2}$ in (30) we get a new integral representation

$$
\operatorname{Erfc}(z)=\frac{z}{\sqrt{\pi}} \int_{1}^{\infty}(\ln t)\left[\frac{2 z^{2}-1}{2 \sqrt{t}}\right] \exp \left(-z^{2} t\right) d t, \quad z>0
$$

of the complementary error function.

COROLlaRY 2.

$$
\frac{1}{a} E_{l}(-a x)=(\ln x) \exp (-a x)-\int_{x}^{\infty}(\ln t) \exp (-a t) d t \quad(x>0, a>0) .
$$

PROOF. This follows from (27) when we take $\alpha=0$ and use the fact [11, page $942(8.359)(1)]$. In particular, when we take $x=1$ in (32), the result [11, page $926(8.212)(16)]$

$$
\frac{1}{a} E_{i}(-a)=-\int_{1}^{\infty}(\ln t) \exp (-a t) d t, \quad a>0,
$$

is recovered.

REMARK. It is interesting to observe that that substitution $a=1$ in (32) yields

$$
E_{i}(-x)=(\ln x) \exp (-x)-\int_{x}^{\infty}(\ln t) \exp (-t) d t
$$

that is, the exponential integral function $E_{i}(-x)$ is equal to the difference of the value of the integrand $(\ln t) \exp (-t)$ at $t=x$ and its integral over the semi-infinite interval $(x, \infty)$.

COROllary 3.

$$
E_{i}(-x) \approx(1+x)(\ln x) \exp (-x)+\gamma \quad \text { as } x \rightarrow 0^{+},
$$

where $\gamma$ is Euler's constant.

PROOF. According to (33) we have

$$
E_{i}(-x)=(\ln x) \exp (-x)-\int_{x}^{\infty}(\ln t) \exp (-t) d t
$$


However (see [11, page 576(4.352)(4)]

$$
\int_{x}^{\infty}(\ln t) \exp (-t) d t=-\gamma-\int_{0}^{x}(\ln t) \exp (-t) d t \quad(x>0),
$$

and according to the first mean value theorem [11, page 211(3.012)] we get

$$
\int_{0}^{x}(\ln t) \exp (-t) d t \approx x(\ln x) \exp (-x) \quad \text { as } x \rightarrow 0^{+} .
$$

Letting $x \rightarrow 0^{+}$in (34) and using (35) - (36) we get the proof of Corollary 3.

\section{Acknowledgements}

The author is indebted to King Fahd University of Petroleum and Minerals for the excellent research facilities and to the referee for helpful comments and suggestions.

\section{References}

[1] M. Aslam Chaudhry and Munir Ahmad, "On a new probability function with applications", to appear.

[2] M. Aslam Chaudhry and Munir Ahmad, "On some infinite integrals involving logarithmic exponential and powers", Proceedings of Royal Society of Edinburgh 122A (1992) 11-15.

[3] A. C. Atkinson, "The simulation of generalized inverse Gaussian, generalized hyperbolic, gamma and related random variables”, Research Report No. 52, Dept Theor. Statist., Aarhus University, 1979.

[4] O. Barndorff-Nielsen, P. Blaesild and C. Halgreen, "First hitting time models for the generalized inverse Gaussian distribution", Stoch. Processes Appl. 7 (1978) 49-54.

[5] B. M. Budak and S. V. Fomin, Multiple integrals, field theory and series (Mir Publishers, Moscow, 1978).

[6] R. S. Chhikara and J. L. Folks, "The inverse gaussian distribution as a lifetime model", Technometrics 19 (1977) 461-468.

[7] R. S. Chhikara and J. L. Folks, "The inverse Gaussian distribution and its statistical application - a review (with discussion)", J. R. Statistic. Soc. B 40 (1978) 263-289.

[8] Erdéyi et al., Higher transcendental functions, Vol. 1 (McGraw-Hill, New York, 1953).

[9] Erdéyi et al., Tables of integral transforms, Vol. 1 (McGraw-Hill, New York, 1954).

[10] I. J. Good, "The population frequencies of species and the estimation of population parameters", Biometrika 40 (1953) 237-60.

[11] I. S. Gradshteyn and I. M. Ryzhik, Tables of integrals, series and products (Academic Press, New York, 1980). 
[12] V. A. Ilyin and E. G. Poznyak, Fundamentals of mathematical analysis (Nauka, Moscow, 1967).

[13] N. L. Johnson and S. Kotz, Distributions in statistics: Continuous univariate distributions 1 (Houghton-Mifflin, Boston, 1970).

[14] B. Jorgensen and B. V. Pedersen, "Contribution to the discussion of O. Barndorff-Nielsen and D. R. Cox: Edgeworth and saddle-point approximations with statistical applications", J. R. Statist. Soc. B 41 (1979) 309-310.

[15] W. John Lee, Well testing (Society of Petroleum Engineers of AIME, New York, 1982).

[16] W. J. Padgett and L. J. Wei, "Estimation for the three-parameter inverse Gaussian distribution", Commun. Statist., - Theor. Meth. A8 (1979) 129-137.

[17] J. J. Shuster, "On the inverse Gaussian distribution function", J. Amer. Statist. Ass. 63 (1968) 1514-1516.

[18] G. N. Watson, A treatise on theory of Bessel functions (Cambridge University Press, Cambridge, 1966).

[19] G. A. Whitmore, "An inverse Gaussian model for labour turnover", J. R. Statist. Soc. A 142 (1979) 468-478.

[20] M. G. Wise, "Skew distributions in biomedicine including some with negative powers of time", in Statistical distributions in scientific work, Vol. 2: model building and model selection (eds. G. P. Patil et al.), (Dordrect Reidel, 1975) 241-262. 